\title{
Más mujeres en los estudios de Informática: una propuesta desde el departamento de formación y orientación laboral
}

\author{
More women in Computer Studies: A Proposal from the Training \\ Department and Career Guidance
}

\author{
Ana Belén Fernández Casado y Marta Ibáñez Pascual'
}

\section{Resumen}

Pese a la relativa paridad entre géneros que se observaba en los albores de la informática, hoy la presencia de mujeres en este tipo de formación es minoritaria. Este hecho, no solo está cerrando una especialización con alta demanda de empleo, también aparta a las mujeres del diseño y construcción del nuevo mundo virtual que nos rodea. La elección educativa de chicos y chicas a lo largo de su itinerario formativo sigue asociada a los estereotipos de género; frente a ello, la escuela no logra atraer y retener a las niñas y chicas en estos estudios, es decir, está fallando en su labor de fomentar la igualdad.

El texto se divide en dos partes: teórica y empírica/aplicada. En la parte teórica se repasan por un lado las razones por las que las chicas no eligen estos estudios; y por otro, nuestra concepción de la Orientación académica y Laboral y los programas que se van implementando para atraer a las chicas a los estudios de informática. En la segunda, y tras explicar el trabajo de campo desarrollado en un Centro de Formación Profesional en el Departamento de Formación y Orientación Laboral, se concreta el objetivo último de estas páginas, los elementos básicos de un programa de atracción y retención de mujeres en estos estudios.

\section{Palabras clave}

Estereotipos, coeducación, profesiones, tecnología informática, buenas prácticas.

\section{Abstract}

Despite the relative parity of gender, observed at the beginning of the computing technology career, today the presence of women in this type of training is a minority. This fact, is not only stopping a specialization with high demand for employment, it also distances the women from the design and construction of the new virtual world that surrounds us. The educational choice of boys and girls throughout their formative years continues to be associated with gender stereotypes; In the face of this, the school is unable to attract and retain young girls in these studies, that is, it is failing to promote equality.

The text is divided into two parts: theoretical and empirical/applied. The theoretical part, reviews the reasons why girls do not choose these studies. The other theoretical support is our concept of Academic and Employment Orientation. In the second part, the Empirical, and after explaining the fieldwork developed in a Vocational Training Center in the Department of Training and Employment Orientation, the information compiled is completed with the others to achieve the ultimate goal of these pages: the basic elements of a program of attraction and retention of women in these studies.

\section{Keywords}

Stereotypes, coeducation, professions, computer technology, good practices. 


\section{Introducción}

La segregación ocupacional persiste con más o menos fuerza en todos los países, de forma que se pueden identificar diversas ocupaciones que permanecen dominadas por uno u otro sexo, siendo una de las causas de la desigualdad entre mujeres y hombres (Hegewisch et al., 2010: 5; Ibáñez, 2008: 94). No solo genera discriminación laboral, también rigideces en el mercado de trabajo y consecuentemente ineficiencia económica (Anker, 1997: 343). En este sentido, la escasa presencia femenina en las ocupaciones informáticas se convierte en problema social por diferentes motivos.

Por una parte, porque la industria tecnológica necesita y necesitará cada vez más profesionales de estos campos, los más demandados en el contexto de la inminente cuarta revolución industrial, y no puede permitirse renunciar al talento femenino. Vivimos en sociedades caracterizadas por la difusión masiva de las tecnologías de la información y la comunicación. La tecnología puntera es ubicua, está presente en nuestros hogares, en los quirófanos y centros de salud, en los aeropuertos e incluso en las tareas del campo (Sainz, 2007: 25).

Además, se subrayan los efectos positivos de saber manejar los ordenadores e Internet (Brynin et al., 2004; Korup y Szydlik, 2005; Liff y Shepperd, 2004; Rogers, 2001). Pues, en su conjunto, este hecho correlaciona positivamente con nuestra capacidad para las relaciones sociales, mejora los resultados escolares, las habilidades matemáticas y de lenguaje y, además, favorece el éxito en la búsqueda de empleo, así como la obtención de salarios más altos (Castaño, Martín y Vázquez, 2008: 139).

Por último, se considera necesario que las niñas y niños aprendan en la escuela a utilizar estas herramientas tecnológicas y de información tanto incorporadas a su proceso general de aprendizaje de distintos saberes, como, sobre todo, en sí mismas, es decir, la escritura de código (Castaño, 2009: 73-74). Sabido es que la frecuencia de uso influye en el conocimiento y el control que se posee acerca de la herramienta $y$, por tanto, en las posibilidades de adaptarla a las necesidades, deseos e intereses de cada persona. Esto tiene efectos importantes desde el punto de vista del diseño de las tecnologías y de sus aplicaciones. Los primeros que utilizan una tecnología son los que contribuyen más directamente a establecer sus usos y, en consecuencia, a modelar su desarrollo. Si son los hombres los que llevan a cabo esta tarea, si las mujeres se quedan fuera, la brecha tecnológica de género aumentará en lugar de reducirse.

La escasa presencia de mujeres en ámbitos técnicos y científicos ha suscitado enorme interés en la comunidad científica a lo largo de los últimos años, tanto a nivel nacional (Elejeibeitia y López-Sáez, 2003; López-Sáez, 1995,) como en el ámbito internacional (Bandura, Barbaranelly, Caprana, y Pastorelli, 2001; Eccles, 1983; Eccles, 2001; Eccles y Wigfield, 2002; Köller, Schnadel y Bäumert 2001; RuizBen, 2003; Stake y Nickens, 2005, entre otros). Estos estudios tienen como denominador común el querer encontrar una explicación al porqué las mujeres, aún con calificaciones escolares equiparables a las de los hombres, siguen desechando estudios relacionados con las matemáticas, la ciencia y la tecnología.

La competencia matemática es la más asociada a las profesiones informáticas. En las pruebas PISA, las puntuaciones entre las chicas de 15 años son ligeramente peores que las de los chicos ${ }^{2}$. Por ejemplo, en España, un 25,1\% de las chicas tienen un bajo rendimiento en matemáticas frente a un 22,1\% de los chicos; tres puntos de diferencia que se reducen a 1,8 en el conjunto de la UE. Sin embargo, aunque mayoritario, este no es un fenómeno universal. En Suecia, Bulgaria, Letonia, Lituania y Finlandia la pro-

2 Se han argumentado diferentes razones y la bibliografía en estos 30 últimos años es ingente. Para una reflexión interesante sobre el tema se puede consultar el informe de la American Association of University Women financiado por The National Science. 
porción de adolescentes varones que puntúan muy bajo en matemáticas es mayor que la de las chicas (en Suecia 28,2 y $26 \%$ respectivamente). (Ver Tabla I en Anexo).

Esta pequeña diferencia se dispara en la elección de estudios posterior. El porcentaje de graduados en ciencias, matemáticas y tecnología en la UE-28 era en 2012 de 37,5 varones del total de graduados, mientras que las mujeres no llegaban al 13\%. Una proporción similar a la española (INE, IneBase tabla-12729). (Una diferencia que no se justifica en el logro en los estudios medios. En la Tabla 1 del anexo se puede apreciar que, de media en la UE, la proporción de mujeres que puntúan bajo en las pruebas PISA en ciencias es menor que la de los varones).

Los datos sobre los índices de presencia femenina en el ámbito educativo nos muestran cómo los roles y estereotipos de género y la elección de trayectorias académicas están interrelacionados (Sainz, 2007: 25). Son, a la vez que un indicador del mantenimiento de dichos estereotipos y desigualdad, una consecuencia o efecto que puede agravar la situación de desigualdad en el futuro. Lo más grave es que las diferencias de género en las profesiones informáticas no parece que tiendan a reducirse en el futuro, ya que son más agudas entre los menores de 40 años que entre los más maduros. Parece que existen barreras invisibles que determinan que la tecnología sea todavía un mundo de hombres, mientras que las mujeres estarían prisioneras de una cierta «tecnofobia» (Castaño, Martín y Vázquez, 2008: 148-149). Este hecho tendrá consecuencias discriminatorias en el futuro laboral de las mujeres y de las jóvenes (Ministerio de Empleo y Seguridad Social, 2013: 3).

El objetivo último de estas páginas es señalar los elementos básicos de un programa de atracción y retención de mujeres en los estudios de informática desde el departamento de Formación y Orientación Laboral (FOL) del Instituto El Bohío de Cartagena, donde una de las autoras desarrolla parte de su actividad profesional. Para ello, el presente texto se divide en dos partes, teórica y empírica, al servicio de sus propios objetivos intermedios. La parte teórica se apoya en dos marcos de reflexión. Por una parte, se analizan las razones por las que las mujeres van perdiendo presencia en estos estudios, y por lo tanto en estas ocupaciones. Por otra, se hace explícita nuestra concepción de la orientación académico-laboral y se hace un somero repaso de algunos de los programas que se van implementando en los últimos años en el entorno español (y latinoamericano). La parte empírica desarrolla un objetivo intermedio de la presente investigación: conocer las opiniones de los agentes interesados sobre los modos de incentivar la matriculación de mujeres en los estudios de informática. En el apartado metodológico se explican las tres fuentes de información utilizadas (encuestas telefónicas semiestructuradas, entrevistas en profundidad y grupo de trabajo de expertos) a lo que se suma la propia observación más informal de la autora como profesora de FOL. El análisis de los resultados empíricos se integra con lo observado en investigaciones ajenas, con idea de completar el objetivo arriba indicado: la propuesta de programa de atracción y retención de mujeres en estos estudios. Se termina con las conclusiones.

\section{Los estereotipos de género, las estudiantes de informática y la Orientación Laboral}

La construcción de la identidad personal está marcada por los estereotipos de género, muy asociados a las elecciones educativas. Los estereotipos vinculados a los roles de «mujer cuidadora»y »hombre mantenedor» seguramente sean los principales. Estos estereotipos se filtran y llegan al alumnado a través de la educación que reciben desde los primeros años de vida; van desde los roles que marcan la vida familiar a las imágenes que impregnan todo lo que les rodea en su vida diaria, en los cuentos y lecturas, en los juguetes, en los libros de texto, en la publicidad, etc. Los niños y niñas manifiestan intereses similares, pero, 
cuando llega el juego simbólico, se refuerza la identidad de género y adoptan como propios los modelos que observan alrededor. Es la etapa donde la representación lúdica será el reflejo simbólico de los roles de varones y mujeres en la sociedad (González, 2009: 111-113)³. Así por ejemplo, carreras como Psicología o Medicina encajan más con la imagen arquetípica de una mujer: persona sensible, que cuida de los demás, que se interesa por aspectos más idílicos y emocionales de su profesión, que es delicada o que se implica en los problemas de los demás (Sainz, 2007: 26).

\subsection{Mujeres en informática, la elección educativa}

Desde la aparición de la informática se ha podido observar un giro en el estereotipo de género asociado a la profesión. Mientras que en su inicio estaba asociado al trabajo de oficina, administrativo y contable, por lo tanto, femenino; en la actualidad, y especialmente entre los jóvenes, es común su asociación con los juegos de ordenador, el hacker, o el friki, asociados a estereotipos masculinos. Como se señala en distintos estudios, mientras que a la mayoría de los chicos les encanta desde pequeños, las chicas tienden a considerarlo como una herramienta, como algo práctico (Castaño, 2009: 86).

Sin embargo, en este proceso de desafección que van sufriendo las mujeres hacia la informática nos encontramos con momentos del ciclo vital especialmente relevantes. La preadolescencia y adolescencia sean seguramente los más importantes ${ }^{4}$.

Sainz establece (2007: 29) que, en un primer momento, la existencia del estereotipo de que las mujeres no son buenas en matemáticas o en ámbitos técnicos fomenta que los padres y los profesores tengan menores expectativas para las chicas que para los chicos, lo cual a su vez reduce la confianza que estas tienen en sí mismas y su posterior rendimiento. Para Eccles y sus colaboradores (Eccles, 1994; Eccles, Barber, y Jozefowicz, 1999; Sainz, 2007: 23) una posible dilucidación a estas diferencias reside en el hecho de que hombres y mujeres asignan distinto valor a esos estudios, fruto del proceso de socialización al que son expuestos a lo largo de las diferentes etapas del ciclo evolutivo. Ya durante la enseñanza primaria los niños discriminan qué asignaturas académicas y qué actividades son más masculinas o femeninas. Por un lado, se asocia la lectura, el arte y la música con las chicas y, por otro lado, las matemáticas, el deporte y las habilidades mecánicas con los chicos (Berk, 1999: 685 en Sainz, 2007: 157-158).

La pérdida de confianza, y con ello de interés, es un elemento clave en este proceso. En general, las mujeres tienden a infravalorar sus habilidades en aquellas ocupaciones tradicionalmente masculinas, incluso en aquellos casos en los que presentan similar desempeño en las pruebas objetivas (Correll, 2004; Hill et al., 2010). En concreto, el círculo vicioso de la pérdida de confianza e interés de las chicas durante los estudios medios y universitarios de informática está suficientemente documentado (Margolis y Fisher, 2002; Castaño, 2009: 86).

Desde la escuela: ¿son las asignaturas presentadas de tal manera que alejan a las chicas? La mala pedagogía en los centros educativos parece que tiene mucho que decir en este círculo vicioso. En primer lugar, en el colegio, los profesores y los orientadores académicos ejercen una influencia considerable y

\footnotetext{
Para un desarrollo exhaustivo ver González (2009).

4 Según un estudio reciente de Microsoft, a los 11 años las chicas tienen el mismo interés por la ciencia y la tecnología que los chicos, pero, en el caso de ellas, empieza a decaer a partir de los 15 años. «Cuando vamos a los colegios nos topamos con una enorme falta de confianza. Nos dicen: 'Estas carreras no serán para mí, no seré capaz de hacerlo, ahí solamente trabajan los buenos'. Estos son mensajes verbalizados por niñas con un potencial enorme, lamenta Pilar López, presidenta de Microsoft España». (El País Semanal (25/06/17).
} 
participan en la consolidación de estereotipos de género existentes y en la activación de las creencias o profecías que se autocumplen, de forma que las expectativas de los profesores sobre sus alumnos se convierten en realidad. En la escuela, los profesores tienen más paciencia con los niños, les dedican más atención, y la cultura informática que se construye es diferente para unos y otras: para los niños, es juego, acción, combate, dominación; para las niñas, chat, listas de correo o club de fans (Castaño, 2009: 87).

Dentro del modelo de expectativa-valor de logro y de elección académica de Eccles y colaboradores (Eccles, 2001), se establece una distinción entre el autoconcepto de habilidad respecto a una tarea específica y el valor que las personas asignan a esa tarea, y se reconoce el importante papel que el autoconcepto tiene en el modelo. Su trabajo se centra en cómo las creencias de competencia de los estudiantes en ciertas actividades -por ejemplo, cómo de buenos se consideran que son para una determinada actividad-, unidas al valor que asignan a una determinada tarea -el interés, el disfrute, la utilidad y la importancia que esa tarea tiene para el individuo- ejercen influencia sobre las elecciones que estos terminan realizando (Sainz, 2007: 35-36).

\subsection{Escuela, coeducación y Orientación Laboral}

Los estereotipos sexistas nos son inculcados desde que nacemos, condicionan la convivencia y las relaciones, y la proyección personal y profesional. En este sentido, la escuela es el reflejo de la sociedad en la que se inserta, y por lo tanto depositaria de estereotipos de género, que transmite desde las primeras etapas de escolaridad y de manera no intencional a través del llamado currículum oculto. Esto incidirá en el futuro desarrollo profesional del alumnado, que sigue respondiendo a los estereotipos sociales diferenciados de las actividades (en especial, estudios y profesiones) según las realicen hombres o mujeres. Si queremos cambios sustanciales en el modelo educativo, es necesario pasar de la escuela mixta a la escuela coeducativa y, para ello, es imprescindible la implicación del profesorado (González, 2009: 111-112).

Los estereotipos de género y la falta de modelos femeninos en este campo son factores influyentes, por lo que diversos estudios apuntan a la necesidad de alentar y motivar a las chicas en edad temprana (Pérez, P. en Dapazo, 2015: 2). La idea subyacente es que la motivación temprana en el conocimiento práctico de las tecnologías informáticas incrementará entre los alumnos del nivel medio su interés por seguir carreras vinculadas con la informática (Dapozo et al. 2014, en Dapazo 2015: 4). Por ejemplo, en una encuesta realizada a 300 alumnos del último ciclo de educación primaria de 12 escuelas de Barcelona y su área metropolitana se observó que las niñas se consideran menos capaces de realizar un programa informático. Iniciando a las niñas en la programación éstas podrán comprobar sus capacidades y por lo tanto mejorar su auto-percepción (Casado et al., 2017: 149).

La Orientación Laboral en las instituciones educativas tiene mucho que decir ante el problema que nos ocupa. Para lograrla, no solo tenemos que hacernos conscientes de dichos estereotipos, también hay que estar atentos a los materiales didácticos con los que trabajan los estudiantes y en general, remover las barreras que contribuyen a que las mujeres tengan una actitud negativa hacia la informática. Los ejemplos españoles en los últimos años son numerosos: los programas en enseñanza primaria en A Coruña (Fernández, 2016: 462) o en Barcelona (Casado et al., 2017: 151), en la idea de que en los años finales de la infancia o principios de la adolescencia (a partir de los 10 años) es cuando niños y niñas empiezan a tener claro el concepto de profesiones y empiezan a desarrollar sus preferencias por 
algunas de ellas (Vondracek -2001- y Hartung et al., -2005). En los niveles intermedios, el programa «Vocaciones en TIC» que tiene como objetivo principal despertar interés en los jóvenes para estudiar carreras vinculadas con las TIC, en forma más amena y desestructurada, mediante la programación de juegos y animaciones, y a través de las actividades propias del quehacer del y la profesional informática (Dapozo et al., 2016: 413). También para alumnado de secundaria la facultad de Informática de la U. de Coruña organizó un concurso, WikinformáticA sobre biografías de mujeres científicas en formato wiki (Ramos et al., 2015, en Calvo y Sanmarco, 2017: 2) asociado al WikiProject Women in Red.

La información y el asesoramiento acerca de las vías de acceso a las carreras tecnológicas no son suficientes para compensar esta visión, ya que muchas chicas creen que hay que ser informático para trabajar en actividades o en empleos informáticos o de Internet, olvidando que la economía, las ciencias empresariales, las humanidades, el diseño o la psicología, son igualmente vías de llegada al sector, especialmente para la creación de contenidos (Castaño, 2009: 87).

Los cambios que se persiguen requieren de una Orientación Laboral con unos objetivos claros y explícitos, que desarrolle programas concretos. Desde una forma diferente de entender la orientación, es decir, como un proceso que va mucho más allá de la elección profesional y que introduce elementos afectivos y relacionales. En este sentido, entendemos la orientación coeducativa, y por lo tanto la forma para atraer y mantener a más mujeres en las aulas de informática (Ministerio de Empleo y Seguridad Social, 2013: 11-13, González, 2009: 113-114). En Educación Secundaria Obligatoria, según las conclusiones de Rodríguez (2015: 554), la tutoría es una parte importante de la acción orientadora dentro de la institución escolar en la que el profesor-tutor es un referente privilegiado como consejeroguía del alumnado. Se dispone de una hora semanal de tutoría para la atención directa al grupo-clase y la concreción del PAT (Plan de Acción Tutorial) y el POAP (Plan de Orientación Académica y Profesional).

La orientación es considerada actualmente parte esencial de la educación, un elemento de calidad, un valor añadido y creciente (Rodríguez, 2008: 65), capaz de mejorar sustancialmente el proceso educativo. Para cumplir con sus objetivos, necesita cumplir dos características: debe ser colectivo y aplicado.

El desarrollo de la orientación es responsabilidad de todos los agentes educativos, convirtiéndose el currículo en la vía natural a través de la cual conseguir los objetivos planteados. Un grupo de miembros del profesorado es capaz de desarrollar las buenas prácticas con mayor efectividad que un docente aislado, y el profesorado respaldado por la directiva, el personal no docente y la comunidad obtendrá todavía mejores resultados. El hecho de cambiar radicalmente la estrategia educativa, propiciando la creación de un ambiente de aprendizaje más acorde a los intereses de los jóvenes y más parecido a su propia realidad hace que los niveles de motivación, rendimiento y autoestima se eleven hasta un punto nunca antes observado (Corchuelo, 2015: 39-40). Para Lola Frutos, docente experta en sociología de la educación y sociología de género «tanto orientadores como profesorado en general son muy importantes porque solamente porque estés a este lado de la tarima, tienes la aureola del saber y siempre estás influyendo y orientando» (XXX, 2017: 206).

5 Para conocer la visión de un grupo de expertos docentes con respecto a la orientación académica y profesional de las mujeres en el ámbito informático ver (XXX, 2017). 
Las «buenas prácticas» docentes no pueden ser rígidas, ni vienen predeterminadas; al contrario, la propia realidad educativa las crea, y el contexto en el que se aplican termina de moldearlas (Corchuelo, 2015: 26). Esa es la razón por la que la propuesta que se presenta aquí nace del trabajo de Orientación Laboral realizado en un contexto concreto: el IES el Bohío en Cartagena.

\section{Un programa de Orientación Laboral aplicado al aumento de las mujeres en los estudios de Informática}

\subsection{Metodología}

Como se indicaba en la introducción de este texto, como objetivo intermedio de la presente investigación se pretende conocer las opiniones de los agentes interesados sobre los modos de incentivar la matriculación de mujeres en los estudios de informática. Para ello se acude a tres técnicas dependientes cronológicamente: entrevistas en profundidad a mujeres trabajando en el sector, un grupo de trabajo con docentes y encuestas telefónicas semiestructuradas a egresados/as. A estas técnicas formalizadas se une la propia experiencia de una de las autoras del texto como profesora de Formación y Orientación Laboral en los ciclos de informática. La experiencia docente de más de once años impartidos en ciclos formativos, siete de ellos en ciclos de la familia profesional de informática, así como siete años de experiencia laboral como técnico de empleo permite a la autora contextualizar y dar sentido a las verbalizaciones de los y las protagonistas.

El Centro en el que nace esta iniciativa es el IES el Bohío. Un centro complejo, con una plantilla que oscila entre 100 y 105 profesores/as, y en el que se oferta el Ciclo de Grado Medio de Sistemas Microinformáticos y Redes (SMR) y el de Grado Superior de Administración de Sistemas Informáticos en Red (ASIR) ${ }^{6}$.

Tabla II. Número de alumnos egresados y encuestados en ASIR el IES El Bohío (201 1-2016)

\begin{tabular}{ccccc}
\hline CURSO & VARONES EGRESADOS & MUJERES EGRESADAS & VARONES ENCUESTADOS & MUJERES ENCUESTADAS \\
\hline $2011-2012$ & 8 & 0 & 7 & 0 \\
\hline $2012-2013$ & 14 & 1 & 11 & 1 \\
\hline $2013-2014$ & 9 & 1 & 8 & 1 \\
\hline $2014-2015$ & 16 & 1 & 11 & 1 \\
\hline $2015-2016$ & 8 & 3 & 7 & 3 \\
\hline TOTAL & $\mathbf{5 5}$ & $\mathbf{6}$ & $\mathbf{4 4}$ & $\mathbf{6}$ \\
\hline
\end{tabular}

Fuente: elaboración propia.

Como se observa en la Tabla II, este último ciclo formativo presenta una alta brecha de género en el Centro (solo el 12\% de los titulados son mujeres); una proporción superior a la de la Región de Murcia, pues en el curso 2014-2015 contaba con 507 alumnos, solo 43 de ellos mujeres ${ }^{7}$ (un 8,5\%).

Las entrevistas en profundidad a doce mujeres que cursaron un ciclo formativo en la familia profesional de informática se llevaron a cabo entre 2012 y 2013, con una duración media de una hora

\footnotetext{
Cuenta también con cuatro cursos de ESO, Programa PMAR en $2 .^{\circ}$ y $3 .^{\circ}$ de la ESO, y un Programa de Refuerzo curricular en $1 .^{\circ}$ de la ESO. Dos cursos de bachillerato, de ciencias y de humanidades y ciencias sociales. Dos Ciclos más de Grado Medio: Cuidados Auxiliares de Enfermería, y Emergencias sanitarias. Y dos Ciclos de Grado Superior: Salud Ambiental, Laboratorio Clínico y Biomédico. (Plan General Anual 2016-2017: https://www.murciaeduca.es/ieselbohio/sitio/ index.cgi?wid_seccion=1)

7 http://econet.carm.es/web/crem/inicio/-/crem/sicrem/PU_EDUCNOUNIV/m14_15/sec41.html
} 
y siguiendo en la medida de lo posible el guión acordado por el grupo de investigación Mujeres en mundos de hombres: razones de la elección formativa, formación, acceso al empleo, trayectoria laboral y vivencia y visión de género (XXX, 2017: 200-202). Estas mujeres fueron seleccionadas buscando las diferentes salidas laborales de la titulación: tres de ellas trabajan en la administración pública y el resto en la empresa privada como asalariadas, excepto una que en el momento de la entrevista estaba desempleada.

Una vez analizadas las entrevistas se realizó el diseño del guión para el grupo de trabajo, que se puso en funcionamiento en 2014. Constó de cuatro informantes clave: dos profesores y una profesora de la familia profesional de informática y una profesora universitaria especialista en sociología del género, que en muchos casos «problematizaba» la visión de los profesores de informática, tendentes a no ver dificultades en la elección de estas titulaciones por parte de las mujeres.

Por último, en mayo del año 2017 se realizaron encuestas semiestructuradas a los alumnos egresados en la familia de informática. Para este ciclo se han entrevistado telefónicamente a 50 alumnos de un total de 61 egresados entre 2012 y 2016. Se disponía de un listado de alumnos ordenado por fecha de obtención del título, y se realizaron dos intentos de localización del alumnado egresado. La duración media de las encuestas fue de 15 minutos, en los que en general se estableció una comunicación cordial y sincera, con un alto grado de rapport, al ser su antigua profesora de Formación y Orientación Laboral quien les realizaba la entrevista.

Entre los 50 alumnos/as del Ciclo de Grado Superior estudiado (ASIR), 6 tituladas son chicas y 44 son chicos. El encuestado más joven tiene 22 años y el más mayor tiene 50 . Además de las cuestiones relacionadas con su inserción laboral, se les ha preguntado sobre la integración de mujeres en el ámbito de la informática y sobre sus gustos y preferencias durante su tiempo de ocio.

El 46\% tenían experiencia previa en el ámbito de la informática, 9 habían cursado un Ciclo de Grado Medio en Sistemas Microinformáticos en Red, y de ellos hay una chica. El 66\% del alumnado está trabajando, y de ellos, el $45 \%$ afirma que sus estudios están relacionados con su trabajo, mientras el $27 \%$ dice que su trabajo no tiene ninguna relación. De los 16 desempleados en busca de empleo, 3 son mujeres, lo que quiere decir que, de las 6 chicas entrevistadas, el 50\% está en el paro.

Los trabajadores en su campo de especialidad son variados: «jefe de informática: programación y administración de sistemas, formación de personal», «realizando las instalaciones a clientes de los equipos, redes», «desarrollador de aplicaciones y administrador de sistemas», «mantenimiento de sistemas, reparación de ordenadores, mantenimiento de instalaciones nuevas, solucionando problemas», $\mathrm{o}$ «mantenimiento informático de red, servidores, página web, la infraestructura de una empresa»y «desarrollador de páginas web y help service», «venta y gestión de dispositivos en tienda».

Hay que decir que en el caso de las chicas, una de ellas trabaja en «reparación y venta de móviles, internet, etc.», otra es dependienta y está estudiando Informática en la universidad. Otra chica es camarera y está estudiando otro ciclo formativo de grado superior en la familia de informática en otro instituto. Las otras tres chicas están desempleadas y no están estudiando. Cuando a ellos les preguntamos en qué tipo de actividad se sitúan las chicas informáticas que ellos conocen, la mayoría afirman que en atención al cliente, aunque también en implantación/resolución de software y reparación de equipos, y también en programación y diseño de páginas web. 


\subsection{Resultados}

Se organiza el análisis a partir de los problemas observados, tanto en la literatura especializada como en nuestra propia investigación (primera columna de la tabla III). Ante los problemas planteados, aparecen las soluciones que se listan en la segunda columna, y que se desarrollan en el presente apartado, (tomando ideas tanto de las fuentes primarias como de las secundarias).

Tabla III. Planteamiento del problema y propuesta de soluciones

\begin{tabular}{|c|c|}
\hline PROBLEMA & SOLUCIÓN \\
\hline \multicolumn{2}{|l|}{ Ámbito general } \\
\hline $\begin{array}{l}\text { Estereotipo } \\
\text { «varón mantenedor» }\end{array}$ & $\begin{array}{l}\text { Normalizar/Visibilizar a las mujeres informáticas: } \\
\text { - Películas y documentales sobre las pioneras. } \\
\text { - Charlas o Jornadas desde la infancia y hasta el bachillerato, de mujeres y hombres en diferentes profesiones, procurando además que en estas representaciones se } \\
\text { - Iuestionen estereotipos. } \\
\text { - Integrar en los contenidos de las materias que damos a nuestro alumnado la historia de las mujeres y su contribución a todas las ramas del pensamiento y las } \\
\text { consecuencias negativas que ha supuesto su invisibilización. }\end{array}$ \\
\hline $\begin{array}{l}\text { Estereotipo } \\
\text { «informático-friki» }\end{array}$ & $\begin{array}{l}\text { Romper los estereotipos con actividades que muestren el amplio espectro de la informática: } \\
\text { - Diseño de páginas web. } \\
\text { - Mantenimiento de bases de datos. } \\
\text { - Seguridad informática. } \\
\text { - Programación. }\end{array}$ \\
\hline $\begin{array}{l}\text { Estereotipo } \\
\text { «informático-varón- } \\
\text { máquina» }\end{array}$ & $\begin{array}{l}\text { Romper el estereotipo de que las máquinas son cosa de hombres: } \\
\text { - Reparación y montaje de equipos durante la ESO } \\
\text { - Campaña de juguetes no sexistas como la de Murcia }\end{array}$ \\
\hline \multicolumn{2}{|l|}{ En el centro } \\
\hline $\begin{array}{l}\text { Escasa proporción } \\
\text { de matrícula }\end{array}$ & $\begin{array}{l}\text { Programa de atracción. Margolis y Fisher (2002): } \\
\text { - Jornadas en la ESO y Bachillerato donde se expliquen los módulos que se imparten en los distintos ciclos de la familia de informática y traer a antiguos alumnos } \\
\text { para que hablen sobre su experiencia formativa y laboral. }\end{array}$ \\
\hline $\begin{array}{l}\text { Problema } \\
\text { de permanencia }\end{array}$ & Buscar la permanencia de las alumnas en estas especialidades con un programa de mentoring. \\
\hline $\begin{array}{l}\text { Ejemplos y proyectos } \\
\text { prácticos próximos al } \\
\text { imaginario masculino }\end{array}$ & Coeducación. Realización de visitas a o por parte de centros donde se hayan implantado proyectos coeducativos. \\
\hline
\end{tabular}

Como se puede apreciar, buena parte de las soluciones están interrelacionadas, su distinción es una mera estrategia analítica. El conjunto de los problemas planteados está reflejado en la literatura, (ver especialmente Blickenstaff -2005-, Brynin, et al., -2004- y Margolis y Fisher -2002). Su organización expositiva (ámbito general y en el centro) es decisión de las autoras, en un deseo de concretar las responsabilidades del IES El Bohío y del departamento de Formación y Orientación Laboral.

Problema general: el estereotipo del varón mantenedor. En este sentido la literatura plantea que el problema en España es similar al de otros contextos internacionales y que responde a una sociedad patriarcal en la que en «esa división histórica del trabajo surgieron dos culturas, una dominante, otra dominada, que se convirtieron en esencias a través de los mitos de lo masculino y lo femenino hasta parecernos lo natural» (Castells y Subirats, 2016: 16).

Frente a la imagen masculina de la informática (y las matemáticas, las ciencias o las ingenierías) potenciar la visibilización de las mujeres informáticas, y por tanto su normalización. Por ejemplo, presentando a la presidenta de Microsoft España, expuesto anteriormente, lo que permite romper con determinados estereotipos que sitúan al hombre como representante de la profesión. El hecho de ver 
imágenes de juguetes relacionándolas con un niño o una niña puede marcar sus gustos y elecciones posteriores 8 .

La falta de visibilización del trabajo de las mujeres en la informática tiene buena solución en los centros educativos. Desde ellos se pueden recomendar materiales extraescolares, como los que plasman los proyectos WikiProject Women in Red y Wikinformática, así como películas y documentales sobre las pioneras, enseñando en las aulas a las mujeres que trabajan en esta materia, muchas de ellas, bien posicionadas. En general, esta ha sido la recomendación más común entre los y las entrevistadas:

E11 (Hombre): Que hagan más películas o más series en las que haya más informáticas protagonistas».

E47 (H): «Algún tipo de demostración de alguna programación que se haya becho por parte de las chicas».

E21 (H): «Ejemplos de mujeres que en el mundo de la informática bayan llegado lejos o que estén bien posicionadas».

E29 (H): «Sería conveniente que cuando se realiza la presentación de los ciclos fuese alguna chica graduada en ese ciclo».

E39 (H): «Enseñando a mujeres que trabajan en esa materia en el instituto, que vean lo que hacen o la reputación a lo mejor que tienen en la empresa o a lo mejor a nivel mundial si son muy buenos o muy conocidos, principalmente enseñando el negocio, y si puede ser que lo enseñe una mujer. Chicas que sepan y que tengan una reputación que enseñen y se vea que se puede vivir de eso sin ningún problema».

E23 (H): «Mostraría fotos de chicas en empresas grandes y haciendo funciones importantes en empresas grandes, que eso boy por hoy no se ve pero si hay. A lo mejor en empresas como GOOGLEy empresas asi grandes, quizá es más parejo lo de chicos y chicas».

Problema general: el estereotipo del informático friki. Este problema se ha detectado tanto en la literatura (Castaño, 2009: 86) como en la investigación propia. Con respecto a los gustos, preferencias y habilidades de los que se dedican a la informática, mayoritariamente los entrevistados consideran que:

E11 (H): «Elperfil de informático suele estar relacionado con un perfil principalmente friki, cultura pop, el manga, comics».

E8 (H): «juegos de ordenador», E12 (H), E17 (H), E20 (H), «videojuegos», E1 (H), E48 (H): «las videoconsolas».

Se podría implementar el programa «Vocaciones» para despertar el interés de los alumnos de la ESO por las TIC. De igual manera, la mayoría de los entrevistados reivindican la normalización de la imagen de la profesión:

8 Bajo la campaña de la Consejería de Familia e Igualdad de Oportunidades de la Región de Murcia, «No condiciones sus juguetes y regala libertad de elección: la imaginación infantil es ilimitada. Contribuye a una sociedad basada en la igualdad», se ha tratado de hacer conscientes a los diferentes agentes sociales la importancia de no transmitir los estereotipos de género a través de los juguetes. Fuente: http://www.igualdadyviolenciadegenero.carm.es/ 
E22 (H): «Jornadas que dieran a conocer un poco más (allá) del estereotipo de lo que es la informática, que es para frikis o algo así, que va mucho más allá de lo que son videojuegos y que tiene otros campos y otras aplicaciones bastante interesantes».

E38 (H): "Sería importante tomarse en serio que la informática y la tecnología la llevamos todos los días todos. Estaría bien aprender normas de seguridad o cosas asi para evitar las cosas que nos podemos encontrar todos los días».

E50 (H): «La gente se tiene que quitar la idea de que es algo para frikis, y que es algo más que los juegos, que hay muchas cosas más por hacer. Yo creo que se tendría que cambiar la visión de que es algo para gente que está todo el día jugando con la consola o el ordenador, que puedes administrar la informática en una empresa, que es una rama muy importante en las empresas y que gracias a él tú puedes solucionar muchas cosas y automatizar muchas cosas dentro de una empresa».

Problema general: el estereotipo del varón-máquina. La gran parte de las indicaciones y propuestas por parte de los entrevistados/as vienen de la mano de explicar mejor los contenidos del trabajo de informático. La enorme variedad de aspectos y habilidades que requiere, a la vez que su interés aplicado. Uno y otro sexo muestran diferentes intereses respecto a los dos grandes campos del trabajo informático (hardware y software): mientras que a los hombres les atrae más el hardware, a las mujeres el software. Ellas están más interesadas en la creación de comunidades y en facilitar las relaciones interpersonales (Castaño, 2009: 85). También nuestros entrevistados así lo señalan:

E19 (Mujer): «Que empezaran a programar muy jóvenes, programar es el futuro».

E20 (H): «La programación les llama más la atención que el hardware y sería un punto a favon».

E28 (H): "Que la informática no es tanto hardware sino que son muchas más cosas. Yo baría un énfasis en eso. Que no es tanto un trabajo manual, sino que es más intelectual de lo que parece a simple vista. Que se enfatice que también se dan bases de datos, programación con bases de datos, seguridad informática también, que se enfaticen esas cosas».

Además, proponen revisar el aspecto de la reparación de equipos:

E49 (H): «En el colegio, si dan informática o tecnología, pues enseñarles lo que es manipular un ordenador, porque yo eso lo veo divertido. Ahora mismo todo el mundo tiene ordenador, o tiene teléfono, tiene algo electrónico. Y enseñarles que si se te rompe lo puedes arreglar así, pues a lo mejor...el que yo misma pueda arreglarlo, no hace falta un hombre, no bace falta una persona aqui que no conozco, a lo mejor puedo bacerlo yo sola... a lo mejor con eso... pueden pensar pues oye me interesa aprender de esto».

E14 (Mujer): "A mi me incentivó la informática el empezar a abrir ordenadores, me entraba la curiosidad. Cursillos para enseñar a abriry el montaje». 
Y, en general, mayor énfasis de todos los aspectos en los primeros años escolares.

E12 (H): «Talleres desde que son chiquititos, algo más de desarrollo de la informática... no tan genérico. No tan enfocados a las chicas hacia la ofimática y a los chicos hacia la reparación y demás. Cuando tienen 10-11 añitos».

E5 (H), E33 (H): «Enseñarles lo que es en el bacbillerato, pues cuando lo conoz̧can se animarán».

Problema en el Centro: escasa proporción de matrícula. Además de comprobarse en las estadísticas publicadas de matriculaciones tanto regionales como nacionales, este es el problema principal detectado en el IES El Bohío. En un centro educativo como el que nos ocupa, con cursos de ESO, se cuenta con una 'población cautiva' para hacer marketing de los estudios de informática. Como se señalaba antes, la Orientación Laboral es un compromiso del conjunto de la comunidad educativa, con el liderazgo de la Dirección y bajo la supervisión técnica del Departamento de Informática, el Departamento de FOL del IES el Bohío está trabajando en un programa que permitirá visibilizar y promocionar los estudios de informática, especialmente entre las chicas y que abarca las propuestas mencionadas anteriormente: despertar el interés de los alumnos de la ESO por las TIC explicando mejor los contenidos del trabajo de informático, revisar el aspecto de la reparación de equipos y de la programación realizando demostraciones y proponiendo talleres para que los alumnos experimenten desde la práctica, e invitando a mujeres informáticas que estén bien posicionadas para que expliquen el trabajo que realizan y sirvan de tutoras de las alumnas que decidan estudiar para evitar el abandono.

Uno de los puntos de inflexión es el último año de la ESO. El alumnado con dificultades durante la ESO suele ser derivado a los ciclos formativos de grado medio que oferte el centro, ya que en la mayor parte de los casos se trata de un alumnado con residencia en el propio entorno9. En el instituto cuyos egresados estamos analizando, con respecto a los Ciclos de Grado Medio se oferta el título de Técnico de Sistemas Microinformáticos, de la familia de informática, mayoritariamente ocupado por chicos y el Ciclo de Grado Medio de Auxiliar de Enfermería, mayoritariamente ocupado por chicas. Nuestro primer esfuerzo se centraría en que durante la ESO se incentive a las chicas que están pensando no seguir por bachiller, a realizar el Ciclo de Grado Medio en SMR. Se debería promover el desarrollo de la auto-percepción de las chicas sobre las cualidades necesarias para el ejercicio de la profesión informática. Valorar, visibilizar y reforzar la idea de que ellas no son solo organizadas y responsables, sino que también poseen las condiciones intelectuales necesarias para desarrollar con éxito la profesión informática (Sánchez y Arias, 2015: 190).

El otro campo de actuación es el bachiller, donde la oferta de asignaturas optativas, seminarios, cursos o charlas puntuales, pueden intentar potenciar el interés de las chicas en las STEM (carreras de ciencia, tecnología, matemáticas e informática) y en concreto en la Informática, pues en los Ciclos Formativos de Grado Superior que oferta su Centro se comprueba que tienen muchas posibilidades de inserción laboral para sus alumnos.

Problema en el Centro: la permanencia. El siguiente paso es la permanencia de estas chicas (y chicos) en la titulación. En este aspecto, el papel de los/las tutores/as del curso es central, especialmente en el caso de las chicas, que pueden verse abrumadas por la mayor presencia de chicos en el

9 Además, a la hora de realizar la orientación, el equipo incide sobre los ciclos ofertados en su propio Centro, asegurando así el mantenimiento de la plantilla docente, sin demérito de la información de los ciclos que se ofertan en otros centros de la zona. 
aula. El tutor/tutora puede incentivar el interés de las chicas por la informática, mostrándola atractiva e interesante, apreciando el valor social que tiene la informática (Sánchez y Arias, 2015: 191-192). Por último, fomentar la posibilidad de continuar los estudios del Ciclo de Grado Superior al alumnado egresado en el Ciclo de Grado Medio aumentando así las oportunidades para la posterior inserción laboral, ya que entre los alumnos de ASIR el porcentaje de inserción es bastante alto. En los Ciclos de Grado Medio la presencia femenina es menor. Aunque son pocas chicas las que se matriculan, la mayor parte de las que inician este tipo de estudios, permanecen y titulan.

Problema en el Centro: una didáctica próxima a la cultura masculina. No parece ser este tampoco un problema en el caso que nos ocupa, es decir, hay una contradicción entre lo que dice la literatura y lo que aparece en la investigación, por lo que habría que investigar con más profundidad en este sentido. En el curso 2016-2017 la plantilla del departamento estaba compuesta por 3 mujeres y 5 hombres. Peso a ello, sigue siendo conveniente que los alumnos/as visibilicen tanto durante la ESO como durante su formación la presencia de las mujeres en la informática, así como durante su proceso formativo en la Formación en el Centro de Trabajo (FCT). Este es un aspecto central, pues gran parte del alumnado que está trabajando, lo hacen en el mismo lugar en que hicieron las prácticas; además, es común que los propios profesores del Departamento de Informática sean los contactos entre egresados y futuros empleadores. El tutor o tutora de la FCT tendrá que asegurarse de que las empresas en las que realizan las prácticas tanto alumnas como alumnos van a permitir que se trabaje en condiciones de igualdad y que tanto las chicas como los chicos puedan tener oportunidades de inserción en el lugar donde hayan realizado sus prácticas.

\section{A modo de conclusiones: reflexión sobre la práctica docente}

El sistema educativo está contribuyendo a la tradicional división del trabajo propio de mujeres y hombres. No por las actuaciones discriminatorias explícitas, sino por la ausencia de análisis y valoración crítica acerca de los estereotipos de género subyacentes y del currículum oculto que se transmite por parte del profesorado. Y, por lo tanto, por la ausencia de acciones curriculares específicas para actuar y operar el cambio. El sistema educativo debe ser agente de este cambio, en este sentido, se deben poner en práctica actuaciones específicamente destinadas a romper con esas barreras mentales que tanto condicionan la vida de nuestras/os jóvenes.

El centro de estudios es el segundo lugar de acceso al ordenador y a Internet, después del hogar familiar, para el 70\% y el 60\% respectivamente de las niñas y niños del grupo de edad entre 10 y 15 años (Castaño, 2009: 75). La escuela puede favorecer el acceso de las féminas a las trayectorias educativas de informática, especialmente removiendo las barreras que están dificultando su acceso. Es un trabajo de la comunidad educativa en su conjunto, liderado desde la Dirección de los Centros y diseñado por el Departamento de Formación y Orientación Laboral. La principal recomendación que podemos hacer tras nuestro análisis de un caso concreto se refiere a la atracción de más mujeres en los estudios de Formación Profesional en la Familia de Informática. Las vías son variadas: visibilizar el papel de las pioneras y a las mujeres exitosas, empezar a programar desde muy jóvenes (a través de talleres o asignaturas optativas de informática en la ESO y Bachillerato), que los alumnos/as también aprendan a reparar equipos, a montar y a desmontar ordenadores, desmontar el estereotipo del informático friki, con la presencia de profesionales del sector tanto femenino como masculino, para que las alumnas también pueda comprender que no hace falta un hombre cuando se trata de utilizar las nuevas tecnologías y no asocien las máquinas con lo masculino. 
Nuestra propuesta de intervención se inicia con el diseño y desarrollo de un modelo de Orientación Laboral, que establece una serie de fases. En una primera fase de exploración se analizará la situación del mercado de trabajo y las posibilidades formativas dentro del ámbito de la informática en el entorno más cercano al IES El Bohío. Para ello se realizará un análisis de las empresas relacionadas con el sector informático entrevistando a los empleadores y en primer lugar a los empresarios que tienen un convenio firmado con el centro educativo para la realización de la FCT.

En una segunda fase, realizaremos un diagnóstico de las competencias requeridas para la inserción laboral de los trabajadores del sector informático que se adquieren dentro de la formación reglada, tanto en la etapa de Educación Secundaria Obligatoria, Bachillerato y durante los Ciclos Formativos específicos de la Familia de Informática. En una tercera fase diseñaremos talleres impartidos por profesorado del centro que permitan adquirir por parte de todo el alumnado de ESO y Bachillerato las competencias que les permitan acceder al mercado de trabajo, incidiendo principalmente en el sector informático, e informándoles sobre las habilidades sociales, los recursos, empresas, instituciones, entre otros que les son necesarios para conseguir una mejor inserción laboral. A los alumnos se les explicarán los contenidos y competencias que pueden adquirir o desarrollar si deciden cursar un Ciclo Formativo de la Familia de Informática tanto de Grado Medio como de Grado Superior.

En una última fase, evaluaremos el resultado de las matriculaciones surgidas después de haber implementado los talleres, para comprobar si ha aumentado el número de mujeres que deciden cursar estudios relacionados con la informática. También se llevarán a cabo Jornadas en las que se pongan en común los resultados de distintas iniciativas en las que se hayan implementado programas de actuación como el propuesto por el IES El Bohío.

El peligro de no hacer nada es que el mercado, tal y como muestran los datos y estadísticas oficiales, nos está llevando a una orientación laboral selectiva y perpetuadora de los roles, donde la mujer tiene las de perder. Para superar la falta de igualdad en el mercado laboral y lograr una elección académica y profesional no estereotipada, es necesario abrir espacios de reflexión y análisis en el profesorado que debe tener en cuenta numerosos aspectos relacionados con el lenguaje, las imágenes y los mensajes que da a su alumnado. Para lograr este cambio, creemos necesario, además de aplicar medidas de sensibilización, formación y análisis en todos los niveles educativos, incidir en los procesos de orientación regulados en la educación secundaria (Ministerio de Empleo y Seguridad Social, 2013: 5-10). Solo desde el compromiso del conjunto de la Comunidad educativa podremos superar este problema que, como ya hemos señalado, está empeorando.

\section{Referencias bibliográficas}

Anker, Richard (1997): "La segregación ocupacional entre hombres y mujeres. Repaso de las teorías". Revista Internacional del Trabajo, 116 (3), 343-370.

Bandura, Albert; Barbaranelly, Claudio; Caprana, Gian Vittorio y Pastorelli, Concetta (2001): "Self-efficacy Beliefs as Shapers of Children's Aspirations and Career Trajectories". Child development, 72 (1), 187-206.

Berk, Laura E. (1999): “Los iguales, los medios de comunicación y la escolarización” en Laura E. Berk. Desarrollo del niño y del adolescente. Madrid: Prentice Hall Iberia. 
Blickenstaff, Jacob Clark (2005): “Women and science careers: leaky pipeline or gender filter?”. Gender and education, 17 (4), 369-386.

Brynin, Malcolm, Raban, Yoel y Soffer, Tall (2004). The New ITCs: Age, Gender and the Family. E-living: Life in a Digital Europe, disponible en http://www.eurescom.de/e-living/

Calvo Iglesias, Encina y Sanmarco Bande, María Teresa (2017): “Más mujeres en Wikipedia”. Perspectiva de xénero na docencia universitaria: IV Xornada Universitaria Galega en Xénero. Santiago de Compostela. Servizo de Publicacións e Intercambio Científico da Universidade de Santiago de Compostela.

Casado Martínez, Carlos; Meneses Naranjo, Julio y Sancho Vinuesa, Teresa (2016): “¿Cómo ven los alumnos de primaria la profesión informática? Influencia del género y la percepción de su capacidad”. Pixel-Bit. Revista de Medios y Educación (en línea). http://www.redalyc.org/articulo.oa?id=36846509011, consultado el 25 de diciembre de 2017. ISSN 1133-8482.

Castaño Collado, Cecilia; Martín Fernández, Juan y Vázquez Cupeiro, Susana (2008): "La e-inclusión y el bienestar social. Una perspectiva de género”. Rev. Economía Industrial, 367, 139-152.

Castaño Collado, Cecilia (2009): “Los usos de Internet en las edades más jóvenes”. Rev. Participación educativa, 11. Consejo Escolar del Estado. Ministerio de Educación. Madrid.

Castells, Manuel y Subirats, Marina (2007). Mujeres y Hombres ¿ Un amor imposible? Madrid: Alianza Editorial.

Corchuelo Fernández, Celia (2015): "Buenas prácticas docentes en contextos de exclusión social”. Cuestiones Pedagógicas, 24, 25-42.

Correll, Shelley J. (2004): "Constraints into Preferences: Gender, Status, and Emerging Career Aspirations”. American Sociological Review, 69, 93-113.

Dapozo, Gladys; Greiner, Cristina; Pedrozo Petrazzini, Gabriel y Chiapello, Jorge (2014): "Vocaciones TIC. ¿Qué tienen en común los alumnos del nivel medio que tienen interés por carreras de Informática?”. IX Congreso de Tecnología en Educación y Educación en Tecnología (TE\&ET 2014), 128-137. Universidad Nacional de Chilecito. Chilecito. La Rioja. 12 y 13 de junio de 2014. ISBN 978-987-24611-1-9

Dapozo, Gladys; Greiner, Cristina; Pedrozo Petrazzini, Gabriel; Chiapello, Jorge; Lencina, Berenice (2015): "Promoción de Vocaciones en TIC. Identificación de factores que influyen en el interés de los alumnos. Promoción de Vocaciones en TIC. Identificación de factores que influyen en el interés de los alumnos". XVII Workshop de Investigadores en Ciencias de la Computación (Salta, 2015). Red de Universidades con Carreras en Informática (RedUNCI).

Dapozo, Glady; Greiner, Cristina; Petris, Raquel; Espíndola, María Cecilia y Company, Ana María (2016): "Promoción del pensamiento computacional para el fomento de vocaciones TIC y mitigación de índices de desgranamiento en carreras de Informática". XVIII Workshop de Investigadores en Ciencias de la Computación (WICC 2016, Entre Ríos, Argentina). Red de Universidades con Carreras en Informática (RedUNCI).

Eccles, Jacquelynne S. (1983): "Expectancies, values and academic behaviors” en Janet T. Spence, Achievement and achievement motives. Psychological and sociological approaches. San Francisco: Freeman and company. 
Eccles, Jacquelynne S., Barber, Bonnie y Jozefowicz, Debra (1999): "Linking gender to educational, occupational and recreational choices: applying the Eccles et al., model of achievement-related choices" en William B. Swann, Judith H. Langlois y Lucia Albino Gilbert (Eds.): Sexism and stereotypes in modern society.Washington: American Psychological Association.

Eccles, Jacquelynne S. (2001): “Achievement” en Judith Worell (Ed.): Encyclopedia of Women and Gender: Sex similarities and differences and the impact of society on gender. San Diego: Academic Press.

Eccles, Jacquelynne S. y Wigfield, Allan (2002): "Motivational beliefs, values and goals". Annual Reviews Psychology, 53, 109-132.

Elejebeita, Carmen y López-Sáez, Mercedes (2003). Trayectorias personales y profesionales de mujeres con estudios tradicionalmente masculinos. Estudio II. Madrid: CIDE/Instituto de la mujer.

XXX, (2017). "Mujeres informáticas. La elección formativa” en Ibáñez et al., 191-222.

Fernández Sánchez, Andrea (2016): "La dirección escolar ante el reto de implantar un plan TIC con perspectiva de género. Investigación joven con perspectiva de género”. Universidad Carlos III de Madrid, Instituto de Estudios de Género, 2016, 462-482.

González López, Isabel (2009): “La orientación académica y profesional en clave de igualdad”. Participación Educativa, 11, 110-121.

Hartung, Paul J.; Porfeli, Erik J. y Vondracek, Fred W. (2005): "Child vocational development: A review and reconsideration”. Journal of vocational behavior, 66 (3), 385-419. http://dx.doi.org/10.1016/j. jvb.2004.05.006

Hegewisch, Ariane et al., (2010). "Separate and Not Equal Gender Segregation in the Labor Market and the Gender Wage Gap”, Institute for Women's Policy Research -IWPR-, Briefing Paper C377. http:/ / www.iwpr.org/publications/pubs/separate-and-not-equal-gender-segregation-in-the-labor-marketand-the-gender-wage-gap, concultado en julio de 2014.

Hill, Catherine, Corbett, Christianne y Rose, Andresse (2010). Why so few? Women in science, technology, engineering, and mathematics. Washington, DC: AAUW (American Association of University Women).

Ibañez Pascual, Marta (2008): “La segregación ocupacional por sexo a examen: características personales, de los puestos y de las empresas asociadas a las ocupaciones masculinas y femeninas". Revista Española de Investigaciones Sociológicas, 123, 87-122.

Ibañez Pascual, Marta (dir.) (2017). Mujeres en mundos de hombres. La segregación ocupacional a través del estudio de casos. Madrid, CIS.

López Sáez, Mercedes (1995). La elección de una carrera típicamente femenina o masculina. Desde una perspectiva psicosocial: la influencia del género. Madrid: Ministerio de Educación y Ciencia.

Korupp, Sylvia y Szydlik, Marc (2005): “Causes and Trends of the Digital Divide”. European Sociological Review, 21 (4), 409-422.

Köller, Olaf, Schnabel, Kai Bäumert, Jürgen (2001): "Does interest matter? The relationship between academic interest and achievement in Mathematics". Journal for Research in Mathematics Education, 32 (5), 448-471. 
Liff, Sonia y Shepherd, Adrian (2004): “An Evolving Gender Digital Divide?”. OII Issue Brief, 2, (en línea). https://www.oii.ox.ac.uk/archive/downloads/publications/IB2all.pdf. Oxford Internet Institute.

Margolis, Jane y Fisher, Allam (2002). Unlocking the clubhouse. Women in Computing. Boston, Massachusetts Institute of Technology.

Ministerio de empleo y Seguridad Social (2013). "Dossier educativo. Segregación de género en las trayectorias formativas y laborales del alumnado". Aula Intercultural. Secretaría general de Inmigración y Emigración, 2 julio 2013. (en línea). http:/ / aulaintercultural.org/materiales/unidades-didacticas-alumnado/

Pérez, Patricia (2015): "Estimular en las niñas el interés por la informática erradicará la desigualdad". Revista Electrónica de Ciencia, Tecnología, Sociedad y Cultura. ISSN 2174-6850. (en línea). https:/ /www.tendencias21.net/Estimular-en-las-ninas-el-interes-por-la-informatica-erradicara-la-desigualdad_a7641. html en Dapozo, Gladys; Greiner, Cristina; Pedrozo Petrazzini, Gabriel; Chiapello, Jorge; Lencina, Berenice (2015): "Promoción de Vocaciones en TIC. Identificación de factores que influyen en el interés de los alumnos. Promoción de Vocaciones en TIC. Identificación de factores que influyen en el interés de los alumnos". XVII Workshop de Investigadores en Ciencias de la Computación (Salta, 2015). Red de Universidades con Carreras en Informática (RedUNCI).

Ramos, Sabela, Ladra, Susana, Freire, Ana, Bolón-Canedo, Verónica, Remeseiro, Beatriz, Andión, José M., \& Castro, Laura M. (2015). "WikinformáticA: visibilización del papel de la mujer en las nuevas tecnologías y promoción de la ingeniería en informática entre las estudiantes de secundaria". Actas de las XXI Jornadas de la Enseñanza Universitaria de la Informática, 113-120. Universitat Oberta La Salle.

Rodríguez Álvarez, Pablo (2015). "La orientación académica y profesional en la enseñanza postobligatoria valorada por el alumnado”. Tesis Doctoral. Universidad de Vigo. (en línea). http://hdl.handle. net/11093/590

Rodríguez Espinar, Sebastián (2008). Manual de tutoría universitaria. (2 ed.). Barcelona: Octaedro. ICE-UB.

Rogers, Everett M. (2001): “The Digital Divide” Convergence,7 (4), 96-111.

Ruiz-Ben, Esther (2003). Looking beyond the software boom: Gendered costs and benefits? En Pasero y Gottburgsen (Hrsg.). Gender from costs to benefits. Wiesbaden: Westdeutscher Verlag.

Sainz Ibáñez, Milagros A. (2007). Aspectos psicosociales de las diferencias de género en actitudes hacia las nuevas tecnologias entre los adolescentes. INJUVE, Madrid.

Sánchez Bello, Ana y Arias Rodríguez, Alicia (2015): "Valoración de la profesión informática en alumnas de educación media: su influencia en la elección de estudios superiores”. Revista de Investigación, 2015, 39 (Mayo-Agosto) (en línea). http://www.redalyc.org/articulo.oa?id=376143541010, consultado el 26 de diciembre de 2017. ISSN 0798-0329.

Stake, Jayne E. y Nickens, Shannon D. (2005): “Adolescent Girls' and Boys' Science Peer Relationships and Perceptions of the Possible Self as Scientist". Sex Roles, 52 (1) (2), 1-11.

Vondracek, Fred W. (2001): “The childhood antecedents of adult careers: Theoretical and empirical considerations” en R. K. Silbereisen \& M. Reitzle (Eds.): Bericht ueberden 42. Kongress der Deutschen Gesellchaft fuer Psychologie in Jena 2000. Lengerich, Germany: Pabst Science Publishers. 


\section{Notas biográficas}

Ana Belén Fernández Casado es Doctora por la Universidad Politécnica de Cartagena. Profesora asociada en el Departamento de Sociología de la Universidad de Murcia e ISEN Centro Universitario y profesora de Formación y Orientación Laboral en la Consejería de Educación de Murcia. Su docencia se ha centrado en el ámbito de la Sociología de la Educación y las líneas de investigación están relacionadas con la formación y la orientación e inserción laboral, género, emprendimiento y exclusión social.

Marta Ibañez Pascual es Profesora Titular del Departamento de Sociología de la Universidad de Oviedo desde el año 2000. Desde 2011 es la Investigadora Principal del Grupo Mujeres en Mundos de Hombres, que analiza las trayectorias laborales de las mujeres en ocupaciones en las que están infrarrepresentadas. Desde 2010 concentra su docencia en la Facultad de Educación de la Universidad de Oviedo.

\section{Anexo}

Tabla I. Porcentaje de bajo rendimiento en lectura, matemáticas y ciencias, por sexo, entre 2009-2012

\begin{tabular}{|c|c|c|c|c|c|c|c|c|c|c|c|c|}
\hline & \multicolumn{4}{|c|}{ LECTURA } & \multicolumn{4}{|c|}{ MATEMÁTICAS } & \multicolumn{4}{|c|}{ CIENCIA } \\
\hline & 2009 & & 2012 & & 2009 & & 2012 & & 2009 & & 2012 & \\
\hline & TOTAL & TOTAL & HOMBRES & MUJERES & TOTAL & TOTAL & HOMBRES & MUJERES & TOTAL & TOTAL & HOMBRES & MUJERES \\
\hline EU & 19,7 & 17,8 & 23,7 & 12,0 & 22,3 & 22,1 & 21,2 & 23,0 & 17,8 & 16,6 & 17,5 & 15,7 \\
\hline Bélgica & 17,7 & 16,1 & 20,8 & 11,5 & 19,1 & 19,0 & 19,3 & 18,5 & 18 & 17,7 & 19,1 & 16,2 \\
\hline Bulgaria & 41,0 & 39,4 & 50,9 & 27,0 & 47,1 & 43,8 & 45,1 & 42,3 & 38,8 & 36,9 & 41,8 & 31,7 \\
\hline República Checa & 23,1 & 16,9 & 22,8 & 10,6 & 22,3 & 21,0 & 19,3 & 22,7 & 17,3 & 13,8 & 14,6 & 12,9 \\
\hline Dinamarca & 15,2 & 14,6 & 19,2 & 10,1 & 17,1 & 16,8 & 15,1 & 18,6 & 16,6 & 16,7 & 16,4 & 17,0 \\
\hline Alemania & 18,5 & 14,5 & 20,1 & 8,7 & 18,6 & 17,7 & 16,8 & 18,7 & 14,8 & 12,2 & 12,9 & 11,5 \\
\hline Estonia & 13,3 & 9,1 & 14,2 & 4,2 & 12,6 & 10,5 & 10,6 & 10,4 & 8,3 & 5,0 & 6,0 & 4,1 \\
\hline Irlanda & 17,2 & 9,6 & 13,0 & 6,1 & 20,8 & 16,9 & 15,2 & 18,7 & 15,2 & 11,1 & 11,6 & 10,6 \\
\hline Grecia & 21,3 & 22,6 & 32,2 & 13,3 & 30,3 & 35,7 & 34,5 & 36,9 & 25,3 & 25,5 & 29,8 & 21,3 \\
\hline España & 19,6 & 18,3 & 23,4 & 13,1 & 23,7 & 23,6 & 22,1 & 25,1 & 18,2 & 15,7 & 15,9 & 15,5 \\
\hline Francia & 19,8 & 18,9 & 25,5 & 12,7 & 22,5 & 22,4 & 22,3 & 22,4 & 19,3 & 18,7 & 20,5 & 17,0 \\
\hline Croacia & 22,4 & 18,7 & 27,3 & 9,5 & 33,2 & 29,9 & 28,8 & 31,0 & 18,5 & 17,3 & 19,5 & 15,0 \\
\hline Italia & 21,0 & 19,5 & 25,9 & 12,6 & 24,9 & 24,7 & 22,8 & 26,7 & 20,6 & 18,7 & 19,6 & 17,8 \\
\hline Chipre & - & 32,8 & 44,5 & 20,5 & - & 42,0 & 42,8 & 41,3 & - & 38,0 & 41,9 & 34,0 \\
\hline Letonia & 17,6 & 17,0 & 25,7 & 8,2 & 22,6 & 19,9 & 21,5 & 18,3 & 14,7 & 12,4 & 15,3 & 9,4 \\
\hline Liłuania & 24,4 & 21,2 & 31,9 & 10,4 & 26,3 & 26,0 & 27,7 & 24,3 & 17,0 & 16,1 & 19,5 & 12,6 \\
\hline Luxemburgo & 26,0 & 22,2 & 26,6 & 17,6 & 23,9 & 24,3 & 20,1 & 28,7 & 23,7 & 22,2 & 20,3 & 24,2 \\
\hline Hungría & 17,6 & 19,7 & 26,9 & 13,0 & 22,3 & 28,1 & 27,6 & 28,5 & 14,1 & 18,0 & 18,8 & 17,2 \\
\hline Malta & 36,3 & - & - & - & 33,7 & - & - & - & 32,5 & - & - & - \\
\hline Holanda & 14,3 & 14,0 & 17,2 & 10,6 & 13,4 & 14,8 & 13,9 & 15,8 & 13,2 & 13,1 & 13,2 & 13,0 \\
\hline Austria & 27,6 & 19,5 & 26,2 & 12,8 & 23,2 & 18,7 & 16,1 & 21,2 & 21,0 & 15,8 & 16,2 & 15,4 \\
\hline Polonia & 15,0 & 10,6 & 16,2 & 5,2 & 20,5 & 14,4 & 15,0 & 13,8 & 13,1 & 9,0 & 10,2 & 7,9 \\
\hline Portugal & 17,6 & 18,8 & 25,0 & 12,5 & 23,7 & 24,9 & 24,0 & 25,9 & 16,5 & 19,0 & 20,3 & 17,7 \\
\hline
\end{tabular}


Más mujeres en los estudios de informática: una propuesta desde el departamento de formación y orientación laboral

\begin{tabular}{|c|c|c|c|c|c|c|c|c|c|c|c|c|}
\hline & \multicolumn{4}{|c|}{ LECTURA } & \multicolumn{4}{|c|}{ MATEMÁTICAS } & \multicolumn{4}{|c|}{ CIENCIA } \\
\hline & 2009 & & 2012 & & 2009 & & 2012 & & 2009 & & 2012 & \\
\hline & TOTAL & TOTAL & HOMBRES & MUJERES & TOTAL & TOTAL & HOMBRES & MUJERES & TOTAL & TOTAL & HOMBRES & MUJERES \\
\hline Rumanía & 40,4 & 37,3 & 46,8 & 28,1 & 47,0 & 40,8 & 40,4 & 41,2 & 41,4 & 37,3 & 39,5 & 35,3 \\
\hline Eslovenia & 21,2 & 21,1 & 30,5 & 11,1 & 20,3 & 20,1 & 20,4 & 19,8 & 14,8 & 12,9 & 14,8 & 10,8 \\
\hline Eslovaquia & 22,2 & 28,2 & 35,4 & 20,4 & 21,0 & 27,5 & 27,6 & 27,3 & 19,3 & 26,9 & 26,8 & 26,9 \\
\hline Finlandia & 8,1 & 11,3 & 17,7 & 4,6 & 7,8 & 12,3 & 14,1 & 10,4 & 6,0 & 7,7 & 9,7 & 5,6 \\
\hline Suecia & 17,4 & 22,7 & 31,3 & 14,0 & 21,1 & 27,1 & 28,2 & 26,0 & 19,1 & 22,2 & 24,8 & 19,6 \\
\hline Reino Unido & 18,4 & 16,6 & 19,8 & 13,5 & 20,2 & 21,8 & 19,7 & 23,8 & 15,0 & 15,0 & 13,9 & 16,0 \\
\hline
\end{tabular}

Fuente: elaboración propia a partir de OCDE (PISA, 2009-2012). 